ESAIM: M2AN 49 (2015) 551-558

DOI: $10.1051 / \mathrm{m} 2 \mathrm{an} / 2014044$
ESAIM: Mathematical Modelling and Numerical Analysis

www.esaim-m2an.org

\title{
FAST AND ACCURATE FINITE ELEMENT APPROXIMATION OF WAVE MAPS INTO SPHERES
}

\author{
SÖREN BARTELS ${ }^{1}$
}

\begin{abstract}
A constraint preserving numerical method for the approximation of wave maps into spheres is presented. The scheme has a second order consistency property and is energy preserving and reversible. Its unconditional convergence to an exact solution is proved. A fixed point iteration allows for a solution of the nonlinear system of equations in each time step under a moderate step size restriction.
\end{abstract}

Mathematics Subject Classification. 65N12, 58J45, 35L70, 35Q75.

Received March 13, 2014. Revised August 14, 2014.

Published online 17 March 2015.

\section{INTRODUCTION}

Wave maps into spheres are weak solutions $u:(0, T) \times \Omega \rightarrow \mathbb{R}^{3}$ of the nonlinear partial differential equation

$$
\partial_{t}^{2} u-\Delta u=\left(|\nabla u|^{2}-\left|\partial_{t} u\right|^{2}\right) u
$$

subject to initial and homogeneous Neumann boundary conditions and the pointwise constraint

$$
|u(t, x)|=1
$$

for almost every $(t, x) \in(0, T) \times \Omega$. The difficulty in their numerical approximation is an appropriate treatment of the constraint and various numerical schemes have recently been proposed. Penalty and projection methods are discussed in [7] and a Lagrange multiplier method is investigated in [8]. Numerical schemes for general pointwise constraints are devised and analyzed in [4]. The methods discussed in those articles satisfy the constraint at the nodes of an underlying triangulation and either require the solution of a nonlinear system of equations in every time step or involve a projection step whose stability leads to restrictive conditions on step sizes or underlying triangulations. An unconditionally stable, linear method has been analyzed in [6] which leads to a violation of the constraint at the order of the step size. A recent development in [10] employs an equivalent first order system that has a symplectic structure which can be preserved by appropriate discretizations. The corresponding spatial finite difference discretization considered therein leads to nonlinear systems of equations in every time step which can be efficiently solved with a fixed-point iteration under a moderate condition on the step size. We show in this article that a similar strategy can be applied for finite element discretizations and

Keywords and phrases. Geometric evolution problem, wave maps, nonlinear partial differential equation, discretization.

1 Abteilung für Angewandte Mathematik, Albert-Ludwigs-Universität Freiburg, Hermann-Herder Str. 10, 79104 Freiburg I.Br., Germany. bartels@mathematik.uni-freiburg.de 
provide a simpler proof for the convergence of the iterative scheme. For related numerical methods we refer the reader to $[1-3,5,12]$ and for analytical aspects of wave maps to $[9,11,13,14]$.

For the special case of the unit sphere considered here an equivalent formulation of the wave map problem can be based on the introduction of the angular momentum $w=\partial_{t} u \times u$ and the identities

$$
\partial_{t} u=u \times w, \quad \partial_{t} w=\Delta u \times u .
$$

Testing the first equation with $u$ shows that this evolution is constraint preserving. Taking the cross product of the first equation with $u$ then yields that $w=\partial_{t} u \times u$. This leads to the identity $\left(\partial_{t}^{2} u-\Delta u\right) \times u=0$ which is a well-known equivalent formulation of the partial differential equation, see $[7,13]$. In [10] it has been proposed to discretize this first-order system in time according to

$$
d_{t} u^{k+1}=u^{k+1 / 2} \times w^{k+1 / 2}, \quad d_{t} w^{k+1}=\Delta u^{k+1 / 2} \times u^{k+1 / 2},
$$

where $d_{t}$ denotes the backward difference quotient for a step size $\tau>0$ and the fractional superscript indicates the average of two successive approximations, i.e.,

$$
d_{t} u^{k+1}=\frac{1}{\tau}\left(u^{k+1}-u^{k}\right), \quad u^{k+1 / 2}=\frac{1}{2}\left(u^{k}+u^{k+1}\right)
$$

with analogous expressions for $w^{k}$. We also apply the backward difference quotient to sequences of real numbers. Testing the discrete system with $u^{k+1 / 2}$ proves the preservation of the constraint, i.e., that

$$
\frac{1}{2} d_{t}\left|u^{k+1}\right|^{2}=d_{t} u^{k+1} \cdot u^{k+1 / 2}=0 .
$$

Testing the discrete system with $\Delta u^{k+1 / 2}$ and $w^{k+1 / 2}$ yields that

$$
\frac{1}{2} d_{t}\left(\left\|\nabla u^{k+1}\right\|^{2}+\left\|w^{k+1}\right\|^{2}\right)=0
$$

where $\|\cdot\|$ denotes the $L^{2}$ norm, i.e., that the total energy is preserved. A corresponding argumentation in a fully discrete setting requires a careful definition of a discrete Laplacian and an appropriate choice of an inner product. With the nodal interpolation operator $\mathcal{I}_{h}$ related to the $P 1$ finite element space $\mathcal{S}^{1}\left(\mathcal{T}_{h}\right)$ on a regular triangulation $\mathcal{T}_{h}$ of $\Omega$ into triangles or tetrahedra we employ the discrete $L^{2}$ inner product $(\cdot, \cdot)_{h}$ defined on $C\left(\bar{\Omega} ; \mathbb{R}^{\ell}\right)$ by

$$
(\phi, \psi)_{h}=\int_{\Omega} \mathcal{I}_{h}[\phi \cdot \psi] \mathrm{d} x=\sum_{z \in \mathcal{N}_{h}} \beta_{z} \phi(z) \cdot \psi(z),
$$

where $\mathcal{N}_{h}$ denotes the set of vertices in $\mathcal{T}_{h}$ with associated nodal basis functions $\left(\varphi_{z}: z \in \mathcal{N}_{h}\right)$ and $\beta_{z}=$ $\left\|\varphi_{z}\right\|_{L^{1}(\Omega)}>0$. The discrete Laplacian $\Delta_{h}: \mathcal{S}^{1}\left(\mathcal{T}_{h}\right) \rightarrow \mathcal{S}^{1}\left(\mathcal{T}_{h}\right)$ related to homogeneous Neumann boundary conditions on $\partial \Omega$ is for $v_{h} \in \mathcal{S}^{1}\left(\mathcal{T}_{h}\right)$ defined by

$$
\left(\Delta_{h} v_{h}, \phi_{h}\right)_{h}=-\left(\nabla v_{h}, \nabla \phi_{h}\right)
$$

for all $\phi_{h} \in \mathcal{S}^{1}\left(\mathcal{T}_{h}\right)^{3}$ with $(\cdot, \cdot)$ denoting the $L^{2}$ inner product. Given $\left(u_{h}^{0}, w_{h}^{0}\right) \in V_{h}$ with $V_{h}=\mathcal{S}^{1}\left(\mathcal{T}_{h}\right)^{3} \times \mathcal{S}^{1}\left(\mathcal{T}_{h}\right)^{3}$ the approximation scheme then consists in computing for $k=1,2, \ldots, K$ approximations $\left(u_{h}^{k}, w_{h}^{k}\right) \in V_{h}$ such that

$$
\begin{aligned}
\left(d_{t} u_{h}^{k+1}, \phi_{h}\right)_{h} & =\left(u_{h}^{k+1 / 2} \times w_{h}^{k+1 / 2}, \phi_{h}\right)_{h}, \\
\left(d_{t} w_{h}^{k+1}, \psi_{h}\right)_{h} & =\left(\Delta_{h} u_{h}^{k+1 / 2} \times u_{h}^{k+1 / 2}, \psi_{h}\right)_{h}
\end{aligned}
$$

for all $\left(\phi_{h}, \psi_{h}\right) \in V_{h}$ and $k=0,1, \ldots, K-1$. This discretization allows us to carry out similar calculations as above which then prove the constraint preservation at the nodes of the triangulation and an energy conservation property, i.e., no discrete dissipation effects occur. Moreover, the discretization has a temporal consistency error of order $\mathcal{O}\left(\tau^{2}\right)$ and is reversible. What remains to be addressed is that discrete solutions exist, that these converge to exact solutions, and that they can be computed reliably and efficiently. 


\section{Constraint And EnERgy PRESERVATion}

We restate the proposed numerical scheme of the introduction.

Algorithm 1. Given $\left(u_{h}^{0}, w_{h}^{0}\right) \in V_{h}$ with $\left|u_{h}^{0}(z)\right|=1$ for all $z \in \mathcal{N}_{h}$ and $\tau>0$ compute a sequence $\left(u_{h}^{k}, w_{h}^{k}\right)_{k=0, \ldots, K} \subset V_{h}$ such that

$$
\begin{aligned}
\left(d_{t} u_{h}^{k+1}, \phi_{h}\right)_{h} & =\left(u_{h}^{k+1 / 2} \times w_{h}^{k+1 / 2}, \phi_{h}\right)_{h}, \\
\left(d_{t} w_{h}^{k+1}, \psi_{h}\right)_{h} & =\left(\Delta_{h} u_{h}^{k+1 / 2} \times u_{h}^{k+1 / 2}, \psi_{h}\right)_{h}
\end{aligned}
$$

for $k=0,1, \ldots, K-1$ and all $\left(\phi_{h}, \psi_{h}\right) \in V_{h}$. The unconditonial existence and boundedness of discrete solutions follows from a fixed-point argument.

Proposition 2.1. Algorithm 1 is feasible and any solution $\left(u_{h}^{k}, w_{h}^{k}\right)_{k=0, \ldots, K}$ satisfies $\left|u_{h}^{k}(z)\right|=1$ for all $k=$ $0,1, \ldots, K$ and $z \in \mathcal{N}_{h}$ and

$$
\frac{1}{2}\left\|\nabla u_{h}^{k+1}\right\|^{2}+\frac{1}{2}\left\|w_{h}^{k+1}\right\|_{h}^{2}=\frac{1}{2}\left\|\nabla u_{h}^{0}\right\|^{2}+\frac{1}{2}\left\|w_{h}^{0}\right\|_{h}^{2}
$$

for $k=0,1, \ldots, K-1$. Moreover, we have $\left\|d_{t} u_{h}^{k+1}\right\|_{h} \leq\left\|w_{h}^{k+1 / 2}\right\|_{h}$ for $k=0,1, \ldots, K-1$.

Proof. (i) Given $\left(u_{h}^{k}, w_{h}^{k}\right) \in V_{h}$ we let $F_{h}^{k}: V_{h} \rightarrow V_{h}$ be defined by $F_{h}^{k}\left(\bar{u}_{h}, \bar{w}_{h}\right)=\left(r_{h}, s_{h}\right)$ with $\left(r_{h}, s_{h}\right) \in V_{h}$ such that

$$
\begin{aligned}
& \left(r_{h}, \phi_{h}\right)=\frac{2}{\tau}\left(\bar{u}_{h}-u_{h}^{k}, \phi_{h}\right)_{h}-\frac{1}{4}\left(\bar{u}_{h} \times \bar{w}_{h}, \phi_{h}\right)_{h}, \\
& \left(s_{h}, \psi_{h}\right)=\frac{2}{\tau}\left(\bar{w}_{h}-w_{h}^{k}, \psi_{h}\right)_{h}-\frac{1}{4}\left(\Delta_{h} \bar{u}_{h} \times \bar{u}_{h}, \psi_{h}\right)_{h}
\end{aligned}
$$

for all $\left(\phi_{h}, \psi_{h}\right) \in V_{h}$. On $V_{h}$ we employ the inner product

$$
\left\langle\left(\alpha_{h}, \beta_{h}\right),\left(\phi_{h}, \psi_{h}\right)\right\rangle_{h}=\left(\alpha_{h}, \phi_{h}\right)_{h}+\left(\nabla \alpha_{h}, \nabla \phi_{h}\right)+\left(\beta_{h}, \psi_{h}\right)_{h} .
$$

With this it follows that

$$
\begin{aligned}
\left\langle F_{h}\left(\bar{u}_{h}, \bar{w}_{h}\right),\left(\bar{u}_{h}, \bar{w}_{h}\right)\right\rangle_{h} & =\left(r_{h}, \bar{u}_{h}\right)_{h}-\left(r_{h}, \Delta_{h} \bar{u}_{h}\right)_{h}+\left(s_{h}, \bar{w}_{h}\right)_{h} \\
& =\frac{2}{\tau}\left(\left\|\bar{u}_{h}\right\|_{h}^{2}-\left(u_{h}^{k}, \bar{u}_{h}\right)_{h}+\left\|\nabla \bar{u}_{h}\right\|^{2}-\left(\nabla u_{h}^{k}, \nabla \bar{u}_{h}\right)+\left\|\bar{w}_{h}\right\|_{h}^{2}-\left(w_{h}^{k}, \bar{w}_{h}\right)\right) .
\end{aligned}
$$

Hence, we have $\left\langle F_{h}^{k}\left(\bar{u}_{h}, \bar{w}_{h}\right),\left(\bar{u}_{h}, \bar{w}_{h}\right)\right\rangle_{h} \geq 0$ for all $\left(\bar{u}_{h}, \bar{w}_{h}\right) \in V_{h}$ such that

$$
\left\|\bar{u}_{h}\right\|_{h}^{2}+\left\|\nabla \bar{u}_{h}\right\|^{2}+\left\|\bar{w}_{h}\right\|_{h}^{2} \geq\left\|u_{h}^{k}\right\|_{h}^{2}+\left\|\nabla u_{h}^{k}\right\|^{2}+\left\|w_{h}^{k}\right\|_{h}^{2} .
$$

Brouwer's fixed point theorem implies the existence of a pair $\left(\bar{u}_{h}, \bar{w}_{h}\right) \in V_{h}$ with $F_{h}^{k}\left(\bar{u}_{h}, \bar{w}_{h}\right)=0$. Defining $u_{h}^{k+1}=2 \bar{u}_{h}-u_{h}^{k}$ and $w_{h}^{k+1}=2 \bar{w}_{h}-w_{h}^{k}$ we find that the equations of Algorithm 1 are satisfied.

(ii) Given $z \in \mathcal{N}_{h}$ the choice $\phi_{h}=u_{h}^{k+1}(z) \varphi_{z} \in \mathcal{S}^{1}\left(\mathcal{T}_{h}\right)^{3}$ in the first equation of Algorithm 1 implies

$$
\frac{\beta_{z}}{2} d_{t}\left|u_{h}^{k+1}(z)\right|^{2}=0
$$

i.e., $\left|u_{h}^{k+1}(z)\right|^{2}=1$ provided that $\left|u_{h}^{0}(z)\right|^{2}=1$. For the choices $\phi_{h}=-\Delta_{h} u_{h}^{k+1 / 2}$ and $\psi_{h}=w_{h}^{k+1}$ we obtain the identities

$$
\begin{aligned}
\frac{1}{2} d_{t}\left\|\nabla u_{h}^{k+1}\right\|^{2} & =-\left(u_{h}^{k+1 / 2} \times w_{h}^{k+1 / 2}, \Delta_{h} u_{h}^{k+1 / 2}\right)_{h}, \\
\frac{1}{2} d_{t}\left\|w_{h}^{k+1}\right\|_{h}^{2} & =\left(\Delta_{h} u_{h}^{k+1 / 2} \times u_{h}^{k+1 / 2}, w_{h}^{k+1 / 2}\right)_{h}
\end{aligned}
$$


and their sum leads to the asserted energy identity. We choose $\phi_{h}=d_{t} u_{h}^{k+1}$ and employ $\left|u_{h}^{k+1 / 2}(z)\right| \leq 1$ for all $z \in \mathcal{N}_{h}$ to estimate

$$
\left\|d_{t} u_{h}^{k+1}\right\|_{h}^{2} \leq\left\|u_{h}^{k+1 / 2}\right\|_{L^{\infty}(\Omega)}\left\|w_{h}^{k+1 / 2}\right\|_{h}\left\|d_{t} u_{h}^{k+1}\right\|_{h}
$$

which yields the bound for $\left\|d_{t} u_{h}^{k+1}\right\|_{h}$.

\section{Remark 2.2.}

(i) For smooth solutions the temporal consistency of the numerical scheme of Algorithm 1 is of second order.

(ii) The scheme is reversible in the sense that the pairs $\left(\widetilde{u}_{h}^{k}, \widetilde{w}_{h}^{k}\right)=\left(u_{h}^{k},-w_{h}^{k}\right)$ satisfy

$$
\begin{aligned}
-\left(d_{t} \widetilde{u}_{h}^{k+1}, \phi_{h}\right)_{h} & =\left(\widetilde{u}_{h}^{k+1 / 2} \times \widetilde{w}_{h}^{k+1 / 2}, \phi_{h}\right)_{h}, \\
-\left(d_{t} \widetilde{w}_{h}^{k+1}, \psi_{h}\right)_{h} & =\left(\Delta_{h} \widetilde{u}_{h}^{k+1 / 2} \times \widetilde{u}_{h}^{k+1 / 2}, \psi_{h}\right)_{h}
\end{aligned}
$$

for $k=0,1, \ldots, K-1$ and all $\left(\phi_{h}, \psi_{h}\right) \in V_{h}$. In case of the CFL condition $\tau \leq c h$ the iterates are uniquely defined ( $c f$. Rem. 4.3 below).

\section{Convergence}

The energy equality of Proposition 2.1 provides a priori bounds on the approximations and implies the existence of accumulation points as the discretization parameters tend to zero. These points define exact solutions of the wave map problem. To prove this we notice the norm equivalence

$$
\left\|v_{h}\right\|_{h} \leq\left\|v_{h}\right\| \leq(d+2)^{1 / 2}\left\|v_{h}\right\|_{h}
$$

for all $v_{h} \in \mathcal{S}^{1}\left(\mathcal{T}_{h}\right)$ and the nodal interpolation estimate

$$
\left|\left(v_{h}, \phi\right)_{h}-\left(v_{h}, \phi\right)\right| \leq c h\left\|v_{h}\right\|\|\phi\|_{H^{2}(\Omega)}
$$

for all $v_{h} \in \mathcal{S}^{1}\left(\mathcal{T}_{h}\right)$ and $\phi \in H^{2}(\Omega)$ with $h>0$ denoting the maximal diameter of elements in $\mathcal{T}_{h}$. We also note that for $\phi \in C(\bar{\Omega})$ we have

$$
\left(v_{h}, \phi\right)_{h}=\left(v_{h}, \mathcal{I}_{h} \phi\right)_{h}
$$

for all $v_{h} \in \mathcal{S}^{1}\left(\mathcal{T}_{h}\right)$. We define piecewise affine and constant interpolants of a sequence $\left(v_{h}^{k}\right)_{k=0, \ldots, K}$ by

$$
\bar{v}_{h}(t, x)=v_{h}^{k+1 / 2}(x), \quad \widehat{v}_{h}(t, x)=\frac{t-t_{k}}{\tau} v_{h}^{k+1}(x)+\frac{t_{k+1}-t}{\tau} v_{h}^{k}(x),
$$

for $t \in\left(t_{k}, t_{k+1}\right]$ with $t_{k}=k \tau$ and for $x \in \Omega$. With these definitions we have for every solution of Algorithm 1 that

$$
\left(\partial_{t} \widehat{u}_{h}, \phi\right)_{h}=\left(\bar{u}_{h} \times \bar{w}_{h}, \phi\right)_{h}, \quad\left(\partial_{t} \widehat{w}_{h}, \psi\right)_{h}=\left(\Delta_{h} \bar{u}_{h} \times \bar{u}_{h}, \psi\right)
$$

for almost every $t \in(0, T)$ and all $\phi, \psi \in C^{\infty}\left([0, T] ; C^{\infty}\left(\bar{\Omega} ; \mathbb{R}^{3}\right)\right)$. With the bounds of Proposition 2.1 we deduce that the inclusions

$$
\begin{aligned}
\widehat{u}_{h} & \in W^{1, \infty}\left(0, T ; L^{2}\left(\Omega ; \mathbb{R}^{3}\right)\right), \\
\widehat{u}_{h}, \bar{u}_{h} & \in L^{\infty}\left(0, T ; H^{1}\left(\Omega ; \mathbb{R}^{3}\right)\right), \\
\widehat{w}_{h}, \bar{w}_{h} & \in L^{\infty}\left(0, T ; L^{2}\left(\Omega ; \mathbb{R}^{3}\right)\right)
\end{aligned}
$$

hold boundedly as $(h, \tau) \rightarrow 0$ and hence there exist accumulation points of appropriate subsequences. These limits are weak solutions of the wave map problem in the sense of the following proposition. For ease of presentation subsequences are not relabeled. 
Proposition 3.1. Assume that $\left(u_{h}^{0}, w_{h}^{0}\right) \rightarrow\left(u_{0}, w_{0}\right)$ in $H^{1}\left(\Omega ; \mathbb{R}^{3}\right) \times L^{2}\left(\Omega ; \mathbb{R}^{3}\right)$ as $h \rightarrow 0$ and that $(u, w) \in$ $L^{\infty}\left(0, T ; H^{1}\left(\Omega ; \mathbb{R}^{3}\right)\right) \times L^{\infty}\left(0, T ; L^{2}\left(\Omega ; \mathbb{R}^{3}\right)\right)$ is such that $u \in W^{1, \infty}\left(0, T ; L^{2}\left(\Omega ; \mathbb{R}^{3}\right)\right)$ and

$$
\begin{aligned}
\widehat{u}_{h} \rightarrow^{*} u & \text { in } W^{1, \infty}\left(0, T ; L^{2}\left(\Omega ; \mathbb{R}^{3}\right)\right), \\
\widehat{u}_{h}, \bar{u}_{h} \rightarrow^{*} u & \text { in } L^{\infty}\left(0, T ; H^{1}\left(\Omega ; \mathbb{R}^{3}\right)\right), \\
\widehat{w}_{h}, \bar{w}_{h} \rightarrow^{*} w & \text { in } L^{\infty}\left(0, T ; L^{2}\left(\Omega ; \mathbb{R}^{3}\right)\right) .
\end{aligned}
$$

Then $|u(t, x)|=1$ for almost every $(t, x) \in(0, T) \times \Omega$ and

$$
\begin{array}{r}
\int_{0}^{T}\left\{\left(u, \partial_{t} \phi\right)+(u \times w, \phi)\right\} \mathrm{d} t=\left(u_{0}, \phi(0)\right), \\
\int_{0}^{T}\left\{\left(w, \partial_{t} \psi\right)+\sum_{j=1}^{d}\left(u \times \partial_{j} u, \partial_{j} \psi\right)\right\} \mathrm{d} t=\left(w_{0}, \psi(0)\right)
\end{array}
$$

for all $\phi, \psi \in C_{c}^{\infty}\left([0, T) ; C^{\infty}\left(\bar{\Omega} ; \mathbb{R}^{3}\right)\right)$ and

$$
\frac{1}{2}\left(\|\nabla u(t)\|^{2}+\|w(t)\|^{2}\right) \leq \frac{1}{2}\left(\left\|\nabla u_{0}\right\|^{2}+\left\|w_{0}\right\|^{2}\right)
$$

for almost every $t \in(0, T)$.

Proof. Owing to the Aubin-Lions lemma we have that $\widehat{u}_{h} \rightarrow u$ in $L^{2}\left(0, T ; L^{2}\left(\Omega ; \mathbb{R}^{3}\right)\right)$ and in particular, that a subsequence converges pointwise almost everywhere to $u$ in $(0, T) \times \Omega$. Let $\phi, \psi \in C_{c}^{\infty}\left([0, T) ; C^{\infty}\left(\bar{\Omega} ; \mathbb{R}^{3}\right)\right)$. An integration by parts and the interpolation estimate for the discrete inner product imply that

$$
\int_{0}^{T}\left\{\left(\widehat{u}_{h}, \partial_{t} \phi\right)+\left(\bar{u}_{h} \times \bar{w}_{h}, \phi\right)\right\} \mathrm{d} t=\left(\widehat{u}_{h}(0), \phi(0)\right)+\mathcal{O}(h)
$$

and a limit passage $(h, \tau) \rightarrow 0$ proves the first identity. For the proof of the second identity we notice that

$$
\begin{aligned}
\left(\Delta_{h} \bar{u}_{h} \times \bar{u}_{h}, \psi\right)_{h} & =\left(\Delta_{h} \bar{u}_{h}, \mathcal{I}_{h}\left[\bar{u}_{h} \times \psi\right]\right)_{h}=-\left(\nabla \bar{u}_{h}, \nabla\left[\bar{u}_{h} \times \psi\right]\right)+\mathcal{O}(h) \\
& =-\sum_{j=1}^{d}\left(\partial_{j} \bar{u}_{h}, \bar{u}_{h} \times \partial_{j} \psi\right)+\mathcal{O}(h)=\sum_{j=1}^{d}\left(\bar{u}_{h} \times \partial_{j} \bar{u}_{h}, \partial_{j} \psi\right)+\mathcal{O}(h) .
\end{aligned}
$$

This identity allows us to pass to the limit in the equation

$$
\int_{0}^{T}\left\{\left(\widehat{w}_{h}, \partial_{t} \psi\right)+\left(\Delta_{h} \bar{u}_{h} \times \bar{u}_{h}, \psi\right)\right\} \mathrm{d} t=\left(\widehat{w}_{h}(0), \psi(0)\right)+\mathcal{O}(h)
$$

and to deduce the asserted equation for $w$. Finally, the energy inequality and the validity of the constraint follow from the available convergence properties.

\section{Remark 3.2.}

(i) The proof shows that we have $u(0)=u_{0}$ continuously in $L^{2}\left(\Omega ; \mathbb{R}^{3}\right)$. Stronger results about the attainment of initial data can be found in [13].

(ii) The employed weak formulation is in fact equivalent to the one used in $[5,7,8]$. This follows from noting that $w=\partial_{t} u \times u$ holds almost everywhere in $(0, T) \times \Omega$ and employing test functions $\psi=u \times \xi$ with appropriate vector fields $\xi$.

\section{ITERATIVE SOLUTION}

We next show that a simple fixed-point iteration allows us to iteratively solve the nonlinear system of equations of Algorithm 1 in every time step. The iterates satisfy the unit-length constraint. 
Algorithm 2. Given $\varepsilon_{\text {stop }}>0,\left(u_{h}^{k}, w_{h}^{k}\right) \in V_{h}$ with $\left|u_{h}^{k}(z)\right|=1$ for all $z \in \mathcal{N}_{h}$ set $\left(u_{h}^{k+1,0}, w_{h}^{k+1,0}\right)=\left(u_{h}^{k}, w_{h}^{k}\right)$ and compute $\left(u_{h}^{k+1, \ell+1}, w_{h}^{k+1, \ell+1}\right)$ for $\ell=0,1, \ldots$ such that

$$
\begin{aligned}
& \left(u_{h}^{k+1, \ell+1}, \phi_{h}\right)_{h}=\left(u_{h}^{k}, \phi_{h}\right)_{h}+\frac{\tau}{4}\left(\left[u_{h}^{k+1, \ell+1}+u_{h}^{k}\right] \times\left[w_{h}^{k+1, \ell}+w_{h}^{k}\right], \phi_{h}\right)_{h}, \\
& \left(w_{h}^{k+1, \ell+1}, \phi_{h}\right)_{h}=\left(w_{h}^{k}, \phi_{h}\right)_{h}+\frac{\tau}{4}\left(\Delta_{h}\left[u_{h}^{k+1, \ell+1}+u_{h}^{k}\right] \times\left[u_{h}^{k+1, \ell+1}+u_{h}^{k}\right], \psi_{h}\right)_{h}
\end{aligned}
$$

for all $\left(\phi_{h}, \psi_{h}\right) \in V_{h}$. Stop the iteration if

$$
\left\|w_{h}^{k+1, \ell+1}-w_{h}^{k+1, \ell}\right\|_{h} \leq \varepsilon_{\text {stop }}
$$

and set $u_{h}^{k+1}=u_{h}^{k+1, \ell+1}$ and $w_{h}^{k+1}=w_{h}^{k+1, \ell+1}$.

\section{Remark 4.1.}

(i) The equations of the algorithm are linear, admit unique solutions, and can be solved successively.

(ii) Any output $\left(u_{h}^{k+1}, w_{h}^{k+1}\right) \in V_{h}$ of the algorithm satisfies

$$
\begin{aligned}
\left(d_{t} u_{h}^{k+1}, \phi_{h}\right)_{h} & =\left(u_{h}^{k+1 / 2} \times w_{h}^{k+1 / 2}, \phi_{h}\right)_{h}+\left(u_{h}^{k+1 / 2} \times r_{h}^{k}, \phi_{h}\right)_{h}, \\
\left(d_{t} w_{h}^{k+1}, \psi_{h}\right)_{h} & =\left(\Delta_{h} u_{h}^{k+1 / 2} \times u_{h}^{k+1 / 2}, \psi_{h}\right)_{h}
\end{aligned}
$$

for all $\left(\phi_{h}, \psi_{h}\right) \in V_{h}$ and with $r_{h}^{k}=w_{h}^{k+1, \ell}-w_{h}^{k+1, \ell+1}$ such that $\left\|r_{h}^{k}\right\|_{h} \leq \varepsilon_{\text {stop }}$.

(iii) Assuming that Algorithm 2 terminates in every time step, unconditional convergence of the corresponding approximations can be proved as $\left(h, \tau, \varepsilon_{\text {stop }}\right) \rightarrow 0$.

To analyze the convergence of Algorithm 2 we notice that with $c_{\mathrm{inv}}>0$ and the minimal mesh-size $h_{\min }>0$ such that the inverse estimate

$$
\left\|\nabla v_{h}\right\| \leq c_{\mathrm{inv}} h_{\min }^{-1}\left\|v_{h}\right\|_{h}
$$

holds for all $v_{h} \in \mathcal{S}^{1}\left(\mathcal{T}_{h}\right)$ we also have

$$
\left\|\Delta_{h} v_{h}\right\|_{h} \leq c_{\mathrm{inv}}^{2} h_{\mathrm{min}}^{-2}\left\|v_{h}\right\|_{h}, \quad\left\|\Delta_{h} v_{h}\right\|_{L^{\infty}(\Omega)} \leq c_{\mathrm{inv}}^{2} h_{\min }^{-2}\left\|v_{h}\right\|_{L^{\infty}(\Omega)}
$$

for all $v_{h} \in \mathcal{S}^{1}\left(\mathcal{T}_{h}\right)$.

Proposition 4.2. Assume that $u_{h}^{k} \in \mathcal{S}^{1}\left(\mathcal{T}_{h}\right)^{3}$ satisfies $\left|u_{h}^{k}(z)\right|=1$ for all $z \in \mathcal{N}_{h}$. Then, the iterates of Algorithm 2 satisfy $\left|u_{h}^{k+1, \ell}(z)\right|^{2}=1$ for all $\ell \geq 0$ and $z \in \mathcal{N}_{h}$. If $c_{\mathrm{inv}} \tau \leq h_{\min }$ then the iteration is a contraction in the sense that for $\ell=1,2, \ldots$ we have

$$
\left\|w_{h}^{k+1, \ell+1}-w_{h}^{k+1, \ell}\right\|_{h} \leq q\left\|w_{h}^{k+1, \ell}-w_{h}^{k+1, \ell-1}\right\|_{h}
$$

with a number $0 \leq q \leq 1 / 2$.

Proof. We abbreviate $\left(u_{h}^{\ell}, w_{h}^{\ell}\right)=\left(u_{h}^{k+1, \ell}, w_{h}^{k+1, \ell}\right)$ and $\left(\widehat{u}_{h}, \widehat{w}_{h}\right)=\left(u_{h}^{k}, w_{h}^{k}\right)$ in this proof. Given $z \in \mathcal{N}_{h}$ the choice $\phi_{h}=\left[u_{h}^{\ell+1}(z)+\widehat{u}_{h}(z)\right] \varphi_{z}$ yields that

$$
\beta_{z}\left(u_{h}^{\ell+1}(z)-\widehat{u}_{h}(z)\right) \cdot\left(u_{h}^{\ell+1}(z)+\widehat{u}_{h}(z)\right)=0
$$

from which we deduce that $\left|u_{h}^{\ell+1}(z)\right|^{2}=\left|\widehat{u}_{h}(z)\right|^{2}=1$. Subtracting the equations that define $u_{h}^{\ell+1}$ and $u_{h}^{\ell}$ leads to

$$
\begin{aligned}
\left(u_{h}^{\ell+1}-u_{h}^{\ell}, \phi_{h}\right)_{h} & =\frac{\tau}{4}\left(\left[u_{h}^{\ell+1}+\widehat{u}_{h}\right] \times\left[w_{h}^{\ell}+\widehat{w}_{h}\right], \phi_{h}\right)_{h}-\frac{\tau}{4}\left(\left[u_{h}^{\ell}+\widehat{u}_{h}\right] \times\left[w_{h}^{\ell-1}+\widehat{w}_{h}\right], \phi_{h}\right)_{h} \\
& =\frac{\tau}{4}\left(\left[u_{h}^{\ell+1}-u_{h}^{\ell}\right] \times\left[w_{h}^{\ell}+\widehat{w}_{h}\right], \phi_{h}\right)_{h}+\frac{\tau}{4}\left(\left[u_{h}^{\ell}+\widehat{u}_{h}\right] \times\left[w_{h}^{\ell}-w_{h}^{\ell-1}\right], \phi_{h}\right)_{h} .
\end{aligned}
$$


Analogously, we subtract the equations that define $w_{h}^{\ell+1}$ and $w_{h}^{\ell}$ to deduce that

$$
\begin{aligned}
\left(w_{h}^{\ell+1}-w_{h}^{\ell}, \psi_{h}\right)_{h} & =\frac{\tau}{4}\left(\Delta_{h}\left[u_{h}^{\ell+1}+\widehat{u}_{h}\right] \times\left[u_{h}^{\ell+1}+\widehat{u}_{h}\right], \psi_{h}\right)_{h}-\frac{\tau}{4}\left(\Delta_{h}\left[u_{h}^{\ell}+\widehat{u}_{h}\right] \times\left[u_{h}^{\ell}+\widehat{u}_{h}\right], \psi_{h}\right)_{h} \\
& =\frac{\tau}{4}\left(\Delta_{h}\left[u_{h}^{\ell+1}-u_{h}^{\ell}\right] \times\left[u_{h}^{\ell+1}+\widehat{u}_{h}\right], \psi_{h}\right)_{h}+\frac{\tau}{4}\left(\Delta_{h}\left[u_{h}^{\ell}+\widehat{u}_{h}\right] \times\left[u_{h}^{\ell+1}-u_{h}^{\ell}\right], \psi_{h}\right)_{h} .
\end{aligned}
$$

The choice $\phi_{h}=u_{h}^{\ell+1}-u_{h}^{\ell}$ and the fact that $\left\|u_{h}^{\ell}+\widehat{u}_{h}\right\|_{L^{\infty}(\Omega)} \leq 2$ allow us to estimate

$$
\left\|u_{h}^{\ell+1}-u_{h}^{\ell}\right\|_{h} \leq \frac{\tau}{2}\left\|w_{h}^{\ell}-w_{h}^{\ell-1}\right\|_{h} .
$$

Similarly, upon choosing $\psi_{h}=w_{h}^{\ell+1}-w_{h}^{\ell}$ we find

$$
\begin{aligned}
\left\|w_{h}^{\ell+1}-w_{h}^{\ell}\right\|_{h} & \leq \frac{\tau}{2}\left\|\Delta_{h}\left[u_{h}^{\ell+1}-u_{h}^{\ell}\right]\right\|_{h}+\frac{\tau}{4}\left\|\Delta_{h}\left[u_{h}^{\ell}+\widehat{u}_{h}\right]\right\|_{L^{\infty}(\Omega)}\left\|u_{h}^{\ell+1}-u_{h}^{\ell}\right\|_{h} \\
& \leq c_{\mathrm{inv}}^{2} \tau h_{\min }^{-2}\left\|u_{h}^{\ell+1}-u_{h}^{\ell}\right\|_{h} .
\end{aligned}
$$

The combination of the last two estimates yields

$$
\left\|w_{h}^{\ell+1}-w_{h}^{\ell}\right\|_{h} \leq \frac{1}{2} c_{\mathrm{inv}}^{2} \tau^{2} h_{\min }^{-2}\left\|w_{h}^{\ell}-w_{h}^{\ell-1}\right\|_{h}
$$

which proves that the iteration is a contraction provided that $c_{\mathrm{inv}} \tau \leq h$.

\section{Remark 4.3.}

(i) If $c_{\text {inv }} \tau \leq h$ and $\varepsilon_{\text {stop }}=h^{\alpha}$ then $N \geq \alpha \log (h) / \log (q)$ steps of Algorithm 2 are required to meet the stopping criterion.

(ii) Proposition 4.2 implies that the iterates of Algorithm 1 are uniquely defined if $c_{\text {inv }} \tau \leq h$.

\section{NUMERICAL EXPERIMENT}

We test the performance of our numerical scheme for initial data from [7] that lead to the formation of a singularity.

Example 5.1. Let $\Omega=(-1 / 2,1 / 2)^{2}, T=1$,

$$
u_{0}(x)= \begin{cases}\frac{1}{a^{2}+|x|^{2}}\left(2 a x_{1}, 2 a x_{2}, a^{2}-|x|^{2}\right) & \text { for }|x| \leq 1 / 2 \\ (0,0,-1) & \text { for }|x| \geq 1 / 2\end{cases}
$$

with $a=(1-2|x|)^{4}$ and $w_{0}(x)=0$ for $x=\left(x_{1}, x_{2}\right) \in \Omega$.

TABLE 1. Maximal iteration number $N_{\ell}$ needed in the fixed-point iterations with stopping criterion $\varepsilon_{\text {stop }}=\tau_{\ell}$ for the discrete time-stepping on a triangulation with mesh-size $h_{\ell}=2^{-\ell}$ and step-size $\tau_{\ell}=h_{\ell} / 4$ for $\ell=3,4, \ldots, 7$ in Example 5.1.

\begin{tabular}{l|lllll}
\hline$h_{\ell}$ & $2^{-3}$ & $2^{-4}$ & $2^{-5}$ & $2^{-6}$ & $2^{-7}$ \\
\hline$N_{\ell}$ & 2 & 2 & 2 & 3 & 3 \\
\hline
\end{tabular}

Our triangulations consist of halved squares with edge-lengths $h_{\ell}=2^{-\ell}, \ell=3,4, \ldots, 7$, and we use the time-step sizes $\tau_{\ell}=h_{\ell} / 4$. The stopping criterion for the fixed-point iteration is chosen as $\varepsilon_{\text {stop }}=\tau$. Table 1 
displays the maximal iteration number needed to achieve the stopping criterion in the fixed-point iteration of Algorithm 2 over all time steps for different refinement levels. We see that this number grows very slowly with the decreasing mesh size $h_{\ell}$ which is in good agreement with our theoretical findings that predict a logarithmic dependence.

Acknowledgements. The author acknowledges the kind hospitality and support from the Isaac Newton Institute for Mathematical Sciences during the research programme Free Boundary Problems and Related Topics.

\section{REFERENCES}

[1] F. Alouges, A new algorithm for computing liquid crystal stable configurations: the harmonic mapping case. SIAM J. Numer. Anal. 34 (1997) 1708-1726.

[2] L. Baňas, A. Prohl and R. Schätzle, Finite element approximations of harmonic map heat flows and wave maps into spheres of nonconstant radii. Numer. Math. 115 (2010) 395-432.

[3] J.W. Barrett, S. Bartels, X. Feng and A. Prohl, A convergent and constraint-preserving finite element method for the $p$-harmonic flow into spheres. SIAM J. Numer. Anal. 45 (2007) 905-927.

[4] S. Bartels, Semi-implicit approximation of wave maps into smooth or convex surfaces. SIAM J. Numer. Anal. 47 (2009) 3486-3506.

[5] S. Bartels, Numerical analysis of a finite element scheme for the approximation of harmonic maps into surfaces. Math. Comput. 79 (2010) 1263-1301.

[6] S. Bartels, Projection-free approximation of geometrically constrained partial differential equations. Preprint (2013).

[7] S. Bartels, X. Feng and A. Prohl, Finite element approximations of wave maps into spheres. SIAM J. Numer. Anal. 46 (2008) $61-87$.

[8] S. Bartels, C. Lubich and A. Prohl, Convergent discretization of heat and wave map flows to spheres using approximate discrete Lagrange multipliers. Math. Comput. 78 (2009) 1269-1292.

[9] A. Freire, S. Müller and M. Struwe, Weak compactness of wave maps and harmonic maps. Ann. Inst. Henri Poincaré Anal. Non Linéaire 15 (1998) 725-754.

[10] T. Karper and F. Weber, A new angular momentum method for computing wave maps into spheres. Technical Report. Preprint ArXiv:1312.3257 (2013).

[11] J. Krieger, W. Schlag and D. Tataru, Renormalization and blow up for charge one equivariant critical wave maps. Invent. Math. 171 (2008) 543-615.

[12] S. Müller and M. Struwe, Spatially discrete wave maps on $(1+2)$-dimensional space-time. Topol. Methods Nonlinear Anal. 11 (1998) 295-320.

[13] J. Shatah and M. Struwe, Geometric wave equations. In vol. 2 Courant Lect. Notes Math. New York University Courant Institute of Mathematical Sciences, New York (1998).

[14] D. Tataru, The wave maps equation. Bull. Amer. Math. Soc. 41 (2004) 185-204 (electronic). 